\title{
Análisis estratégico sobre la migración del talento en el ámbito de la Unión Europea
}

\author{
Marta Ferrer Serrano \\ Departamento de DiREcción Y Organización de EMPRESAS \\ UNIVERSIDAD DE ZARAGOZA \\ martafe@unizar.es \\ María Pilar Latorre Martínez \\ Departamento de Dirección y Organización de Empresas \\ UNIVERSIDAD DE ZARAGOZA \\ latorre@unizar.es \\ Ramón Hermoso Traba \\ DePARTAMENTO de INFORMÁtICA E INGENIERIA DE SistemaS \\ UNIVERSIDAD DE ZARAGOZA \\ rhermoso@unizar.es
}

\section{Resumen:}

Introducción: Este artículo se centra, en primera instancia, en poner de relieve la importancia que tiene la movilidad de los recursos humanos y en concreto de talento 
en la Unión Europea, bajo la premisa de que este fenómeno en la actualidad continúa intensificándose, debido a la profunda competencia que existe.

Metodología: La parte central del trabajo consiste en la recopilación de datos desde dos perspectivas diferentes: económica y educativa. A continuación, se procede al análisis de dichos datos cuantitativos a través de correlaciones estadísticas (coeficiente de Pearson).

Resultados: Se obtiene una serie de resultados que ponen de manifiesto la relación existente entre las variables objeto de estudio. En concreto, se hayan distintas correlaciones estadísticas significativas.

Conclusión: La interrelación de las variables macroeconómicas y educativas seleccionadas en este estudio aportan información relevante que pone de manifiesto el panorama general de la movilidad del talento de la Unión Europea dejando ver los nuevos retos a los que se enfrenta de manera general y de manera individual los países miembros.

Palabras clave: migración, movilidad, talento, Unión Europea, población.

\section{Strategic analysis of the migration of talent within the European Union}

\section{Abstract:}

Introduction: this article focuses primarily on highlighting the importance of the mobility of human resources, especially talent, within the European Union. This is approached from the premise that the phenomenon is becoming ever more prevalent in today's world due to the presence of a deeprooted sense of competition.

Methodology: a large part of the article is devoted to the collection of data from two different perspectives: economics and education. The quantitative data is then analysed using statistical correlations (Pearson's coefficient).

Results: there are a number of findings that draw attention to the relationship between the variables forming the object of this study, particularly in the case where any significant statistical correlations emerge.

Conclusion: the relationship between the macroeconomic and educational variables selected in this study throw up relevant information that reveals the general situation of the mobility of EU talent, providing a glimpse of the new challenges - both general and individual - that the member states need to tackle.

Key words: migration, mobility, talent, European Union, population. 


\section{Análisis estratégico sobre la migración del talento en el ámbito de la}

Unión Europea

Marta

Ferrer Serrano

María Pilar

Latorre Martínez

https://doi.org/10.26754/ojs_ais/ais.2019404201

Ramón

Hermoso Traba

\section{INTRODUCCIÓN}

La motivación que impulsa el desarrollo de este artículo se extiende al interés que suscita esta temática, sobre todo desde que la crisis económica está presente en todo el territorio europeo. Por ello, se realiza una radiografía exhaustiva y crítica del flujo de talento y de la migración del capital humano en la Unión Europea, en adelante UE, así como de la interrelación existente entre esta movilidad y el nivel de estos países desde tres perspectivas diferentes: social, económica y educativa.

La importancia de este proyecto radica en que "los recursos humanos juegan un papel central en la producción de conocimiento y, por lo tanto, en el desarrollo tecnológico y económico de los países (OCDE, 2008). Además, de acuerdo con este organismo, la intensidad del crecimiento del conocimiento implica una mayor necesidad de especialistas altamente cualificados que sean capaces de "acceder, entender y usar el conocimiento" (OCDE, 2008).

En cambio, a pesar de que la crisis ha intensificado este fenómeno migratorio, la movilidad internacional del trabajo no es un fenómeno nuevo (Harris y Todaro, 1970). Las personas se han movido siempre a otros países en busca de mejores perspectivas económicas, para escapar de conflictos o por motivos 
vinculados a la reunificación del núcleo familiar (Nadeen y SeJik, 1995).

Sin embargo, en los últimos años, este movimiento, se ha visto potenciado como resultado de la globalización de la actividad económica, los mecanismos de mercado que incrementan la actividad comercial y la crisis económica con la correspondiente escasez de mano de obra adecuada a las necesidades que se demandan en momentos determinados (OCDE, 2008).

Por todo esto, atraer la inmigración más cualificada es un deseo compartido a escala global (en la literatura conocido como "global race for talent") (Lewin et al., 2009; Sachar, 2006). En esta competición, todos los países buscan incrementar su atractivo para atraer a los individuos más brillantes.

Hoy en día, la movilidad internacional por motivos laborales se considera una cuestión de gran relevancia que suscita controversia entre los países emisores, en cuanto a la pérdida de inversión realizada en el talento emigrante (Puri, 2008). En concreto, no existen estudios actuales sobre la migración de talento en la UE, al ser una problemática actual acentuada por la crisis sufrida en los últimos años. Es en este punto donde se encuentra el interés de nuestro estudio y el vacío en la literatura académica.

El objetivo general de este trabajo es analizar estratégicamente la movilidad del talento en la Unión Europea teniendo en cuenta la perspectiva educativa y económica y proporcionando una imagen panorámica de la situación. Para ello, se realiza un estudio bibliográfico profundo y se analizan las variables a partir de correlaciones estadísticas.

La estructura que sigue este artículo es la siguiente. En la primera sección se fundamenta teóricamente el estudio en base realizándose una búsqueda de documentación bibliográfica sobre los flujos migratorios en la UE. A continuación, se realiza un examen exhaustivo en relación con los veintiocho países que componen la UE y los diferentes indicadores escogidos. La siguiente sección consiste en la realización del análisis estadístico de las variables. Por último, se presentan las conclusiones del estudio.

${ }^{1}$ Para más información acudir al libro de Anna Buoucher "Gender, migration and global race for talent”, Manchester University Press (2016). 


\section{MARCO TEÓRICO}

La relación entre migración y desarrollo ${ }^{2}$ ha sido motivo de debate en numerosos artículos y libros especializados (De Haas, 2010). Los efectos de la migración en los países de origen han variado según los distintos autores y corrientes, aunque el trabajo empírico sobre la movilidad ha sido menor que el relativo a las consecuencias de la migración para los países receptores (Pellegrino, 2000).

A la hora de extraer conclusiones, Papademetriou y Martin (1991), tras investigar y analizar diferentes áreas de estudio, concluyeron que la relación entre migración y desarrollo debía considerarse como "no resuelta"3" (Appleyard, 1992), y que, por lo tanto, era difícil extraer principios generales ya que, a su juicio, las ventajas y desventajas variaban mucho en función de los casos analizados. Al tratarse de una cuestión no resuelta, las respuestas no son las mismas para todas las situaciones, y las conclusiones de los estudios varían dependiendo de los factores que se consideren y del punto de vista que se adopte.

La literatura ha tratado de manera individual los factores que provocan los movimientos de las personas y que conforman el desarrollo (Savage y Egerton, 1997; South y Deane, 1993). Sin embargo, en la actualidad existe gran interés por analizar de qué modo se relacionan y qué ventajas supone la movilidad de la población para los distintos ámbitos (De Haas, 2010).

Los autores coinciden en sus estudios en que el efecto más destacable que produce la movilidad de los recursos humanos (aunque su estimación resulta compleja), es el aumento del Producto Interior Bruto, en adelante PIB, de los países de acogida (Edwards, 2001). Otro de los beneficios que conlleva la migración poblacional, es el enriquecimiento cultural y de conocimientos que se produce al cruzar los distintos límites fronterizos (Canales y Zlolniski, 2001). De acuerdo con Canales y Zlolniski (2001), a pesar de estas ventajas, el peso de los inconvenientes (sobre todo para los países de origen) es mucho mayor.

${ }^{2}$ Se entiende por desarrollo el crecimiento económico, social, cultura y tecnológico de un territorio o área determinada.

${ }^{3}$ En inglés, "the unsettled relationship". El concepto aparece descrito y estudiado teóricamente en "Migration and Development: A Global Agenda for the Future", de R. T. Appleyard, 1992. 
La globalización en relación con la movilidad de recursos humanos ha traído consigo desde una perspectiva nacional una pérdida de competitividad comparable a otros países importadores de talento, y desde una perspectiva más individualista, un desarraigo al país de origen y al núcleo familiar (Templer et al., 2006). Esto provoca un envejecimiento de la población para los países que soportan gran tasa de emigración dado que, de manera mayoritaria, el éxodo se produce en edades comprendidas entre los 20 y 40 años ${ }^{4}$.

Además, de acuerdo con Mahroum (2000), el mercado de trabajo está cada día más globalizado sobre todo para el personal altamente cualificado. La contrapartida implica que las organizaciones están dispuestas a invertir cada vez más en personal altamente cualificado y que, a su vez, puedan disponer de movilidad internacional y que aseguren un rendimiento de alta calidad, así como eficiencia (Mahroum, 2000).

Esta alta cualificación, el desarrollo de los países y las variables coyunturales del entorno, suponen la reorganización y movilidad del personal altamente cualificado en busca de nuevas oportunidades de futuro (Templer et al., 2006). Los países, sin darse cuenta, se ven envueltos en una profunda guerra por el talento, para que, así como consecuencia, su competitividad, a nivel organizacional, y su posición relativa, tanto a nivel organizacional como a nivel nacional, aumenten. Es de esta manera cuando surge el concepto de "fuga de cerebros" o "brain drain ${ }^{5 "}$.

El concepto hace referencia a la salida de población con una alta cualificación o nivel de estudios (profesionales, técnicos y científicos entre otros), de un país o sectores económicos, hacia otro en búsqueda de unas mejores condiciones laborales o de autodesarrollo personal (Brandi, 2006). Generalmente, la fuga de cerebros hace perder a un país la inversión hecha en la educación superior de la persona que decide emigrar (Brandi, 2006). Entre las principales causas destaca la falta de oportunidades de

${ }^{4}$ De acuerdo con Eurostat, la edad media de los inmigrantes en la UE en 2014 era de 28 años. Página web: http://ec.europa.eu/eurostat/statistics-explained/ index.php/Migration_and_migrant_population_statistics/es\#Flujos_migratorios

${ }^{5}$ En Gaillard y Gaillard (1998) se explica la historia del concepto "brain drain". 
empleo, el mal ambiente social y laboral y la falta de apoyo a los estudiantes recién graduados (Miyagiwa, 1991).

Por tanto, la preocupación por la pérdida de recursos humanos cualificados no es un hecho nuevo, si bien, en una esfera mundial, es en las décadas de 1950 y 1960 (a partir del fin de la Segunda Guerra Mundial, y paralelamente con la intensificación del proceso de migración en África, América del Sur y Asia), cuando adquiere relevancia en el ámbito académico y en los debates de los organismos internacionales (Brandi, 2006). "Además, la segunda mitad del siglo XX certificó la conversión de la Europa Occidental en una zona de recepción de inmigración. Esa inversión en el sentido de los movimientos migratorios estuvo estrechamente relacionada con las transformaciones demográficas, económicas y políticas de Europa" (Cabré y Domingo, 2002).

Por otro lado, siguiendo a los autores Cabré y Domingo (2002), la crisis económica de los años setenta tuvo una especial repercusión en la ocupación en el sector industrial, y denotó importantes consecuencias en los movimientos migratorios y en las políticas dirigidas tanto a la migración como a la población extranjera residente en cada uno de los países que habían destacado como receptores de inmigración. "Una de las primeras medidas adoptadas para combatir el creciente desempleo fue orientada a la restricción de la llegada de nuevos flujos migratorios, a la vez que se incentivaba el retorno de los inmigrantes residentes. (...) Mientras que para los países del sur de Europa el retorno de inmigrantes fue una realidad, para el resto de las nacionalidades ese retorno no sólo no se produjo en el volumen deseado, sino que al final del período el número de inmigrantes procedentes de países terceros a la Unión Europea había crecido. (...) El aumento de menores y mujeres puso de manifiesto la voluntad de asentamiento de la inmigración, acabando con el modelo del trabajador inmigrante identificado exclusivamente con jóvenes de sexo masculino" (Cabré y Domingo, 2002).

En la década de los noventa, los acuerdos firmados en Luxemburgo permitieron a los ciudadanos europeos viajar sin tener que presentar el pasaporte en las fronteras. Millones de jóvenes comenzaron a estudiar en otros países. Además, se acuerda en 1999 como nueva moneda europea común, el 
Euro $(€)$ (Europa.eu, 2016). Se facilita de esta manera el intercambio de flujos poblacionales de los países miembros pertenecientes a la UE.

Las tendencias migratorias de los Estados Miembros de la UE cambiaron a lo largo de estos años. La reactivación económica de los ochenta, la desintegración del bloque soviético y la caída del muro, se tradujeron en el aumento de los flujos migratorios hacia toda Europa occidental, con especial fuerza en los primeros años de la década de los 90 . Además, los nuevos países incorporados y convertidos en la frontera sur de la UE presentan una inmigración de carácter principalmente económica (Delgado, 2002).

La apertura gradual de los mercados laborales europeos occidentales por las ampliaciones de 2004 y 2007 de la UE, permitió a muchos trabajadores salir a buscar empleo y nuevos proyectos en la zona más próspera de la UE. Este hecho cambió drásticamente el panorama de la migración en Europa y condujo a flujos migratorios sustancialmente de este a oeste (Kahanec, 2012).

Como ya se ha señalado, la década de los 90 destacó por la aceleración de los movimientos migratorios. Por otro lado, es característico al conjunto de los países de la UE, el protagonismo que el saldo migratorio adquiere en el cómputo global del crecimiento de la población. La creciente llegada de flujos migratorios juntamente con el papel esencial que juegan en el crecimiento total de la población de la UE, hizo que en la década de los 90 se plantee el diseño de una política migratoria conjunta (Cabré y Domingo, 2002).

En septiembre de 2008 la crisis financiera sacude la economía mundial provocando un aumento masivo de las migraciones poblacionales. Asimismo, aumentan las revueltas que llevan a muchas personas a huir de sus hogares y buscar refugio en Europa. La UE se enfrenta al dilema de qué políticas establecer para la regulación de este escenario (Europa.eu, 2016). Por otro lado, como consecuencia de la profunda crisis que está soportando Europa, un gran número de población se encuentra en una situación de inactividad en el empleo trayendo consigo la búsqueda de nuevas oportunidades de futuro con independencia del territorio. 
La literatura más reciente apunta como los motivos de movilidad están cambiando. Según Krishnan y Kirubamoorthy (2017) en los últimos tiempos, el patrón ha cambiado a una tendencia creciente de expatriación autoiniciada que cubre un grupo diverso de individuos de diversos antecedentes y disciplinas (Global Mobility Trends Survey, 2016). También estamos viendo el surgimiento de la categoría llamada carrera proteica (Hall y Moss, 1998). La carrera proteica es un proceso en el cual la persona y no la organización está manejando la carrera, lo que incluye las variadas experiencias de la persona en educación, capacitación, trabajo en varias organizaciones, cambios en el campo ocupacional, etc. La persona proteica hace elecciones personales de carrera y busca la autorrealización haciéndose cargo de sus objetivos futuros, siendo el criterio del éxito un factor intrínseco (éxito psicológico).

En la siguiente sección se presenta la muestra, variables y fuentes de información, así como la metodología llevada a cabo en este estudio.

\section{METODOLOGÍA}

\section{Muestra y variables}

Este artículo se ha basado en la obtención y recopilación de datos cuantitativos de la migración poblacional y más concretamente, del talento en la UE desde dos perspectivas (económica y educativa) en el año 2015. Dicha recopilación de información se ha realizado utilizando fuentes de información secundarias, en concreto, se ha recurrido a bases de datos oficiales como Eurostat, AMECO (base de datos macroeconómica anual de la Dirección General de Asuntos Económicos y Financieros de la Comisión Europea), los Institutos Nacionales de Estadística de cada país miembro de la UE, la Comisión Europea, y la OCDE (Organización para la Cooperación y el Desarrollo Económico).

La muestra de este trabajo consta de la población de los 28 países pertenecientes a la Unión Europea. Las variables analizadas incluyen únicamente a ciudadanos con nacionalidad en los países europeos y registrados de alguna manera en dichos 
países y por tanto en las bases de datos. Las variables objeto de estudio son las siguientes:

\section{Variables dependientes:}

- Tasa de inmigración: ratio entre el número total de inmigrantes y la población total registrada de cada país.

- Tasa de emigración: ratio entre el número total de emigrantes y la población total registrada de cada país.

Variables independientes:

- Tasa de desempleo: número de desempleados dividido por la población activa, y se expresa en forma de porcentaje. Esta variable se desagrega según género.

- Participantes programa Erasmus+.

- Subsidio por individuo participante en el programa Erasmus +: Cuantía total destinada por cada país para el programa Erasmus + dividido entre el número de participantes total de cada país.

- Gasto público educación: Partida del presupuesto destinado por cada Gobierno con razones educativas.

- PIB pc: Indicador económico que mide la relación existente entre el nivel de renta de un país y su población. Para ello, se divide el Producto Interior Bruto de dicho territorio entre el número de habitantes.

- Importaciones y exportaciones: Saldo comercial en términos de exportaciones y de importaciones.

- Graduados niveles educativos: Número total de población que ha obtenido determinado grado educativo. En concreto se consideran los estudios obligatorios (hasta 16 años, segundo curso de la ESO), la secundaria no obligatoria (hasta 18 años, 
bachillerato), grados universitarios, máster y programas de doctorado.

- Tasa de ofertas de empleo por sectores: Porcentaje representativo de la tasa de ofertas de puestos de trabajo por sectores de actividad para cada país.

\section{Técnica metodológica}

El análisis estadístico se ha llevado a cabo a través del software informático "IBM SPSS Statistics 23" y en concreto en su función de correlación bivariable (centrando el análisis en el coeficiente de correlación de Pearson). El coeficiente de correlación de Pearson viene definido por la siguiente expresión:

$$
R_{x y}
$$

La interpretación del coeficiente de Pearson es la siguiente: "el valor de la correlación es igual a 1 o -1 si la correlación es de intensidad máxima, y se va acercando hacia el 0 cuanto menor sea la intensidad de la correlación. Además, el índice tiene signo positivo cuando la correlación es directa y negativo cuando es inversa" (Chorro, 2016).

\section{RESULTADOS}

A continuación, se pasa a analizar estadística y analíticamente las variables seleccionadas. En la Tabla 1 se recogen las matrices de correlaciones elaboradas y posteriormente se exponen los resultados más significativos al respecto. 


\section{Tabla 1}

\section{Matrices de correlaciones}

\begin{tabular}{|c|c|c|}
\hline 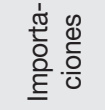 & 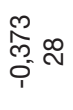 & $\stackrel{L}{0}_{0}^{\infty} \stackrel{\infty}{\sim}$ \\
\hline 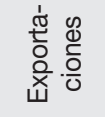 & $\begin{array}{l}\hat{N} \\
\text { ల్ } \\
\text { i }\end{array}$ & $\underset{O}{\tilde{O}} \stackrel{\infty}{\sim}$ \\
\hline 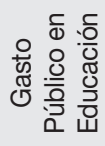 & $\underset{\substack{\hat{O} \\
\sigma^{-}}}{\stackrel{\infty}{N}}$ & $\stackrel{+}{\sigma}_{\sigma_{-}^{*}}^{\infty} \stackrel{\infty}{\sim}$ \\
\hline 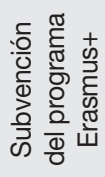 & $\begin{array}{l}\stackrel{*}{m} \\
\stackrel{\sim}{+} \stackrel{\infty}{\sim} \\
\stackrel{i}{i}\end{array}$ & $\frac{\hat{N}}{\sigma_{i}} \stackrel{\infty}{\sim}$ \\
\hline 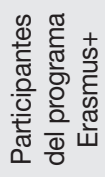 & 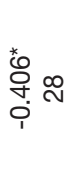 & $\frac{\sim}{\sim} \underset{i}{\sim} \stackrel{\sim}{\sim}$ \\
\hline 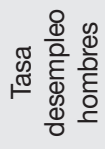 & 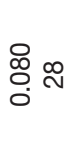 & $\begin{array}{l}\text { స్ } \\
\text { ஸ̊ } \\
\text { i }\end{array}$ \\
\hline 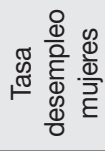 & $\underset{\delta}{\bar{\delta}} \stackrel{\infty}{\sim}$ & 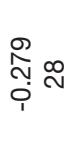 \\
\hline \multirow[t]{2}{*}{$\begin{array}{l}\stackrel{0}{\alpha} \\
\frac{m}{\alpha}\end{array}$} & 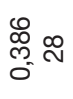 & 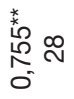 \\
\hline & 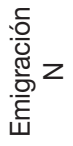 & 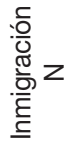 \\
\hline
\end{tabular}

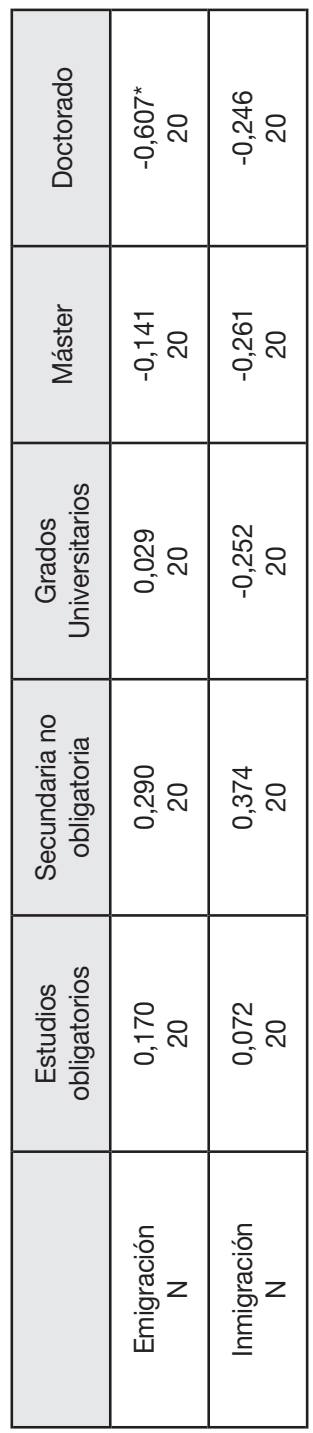

\begin{tabular}{|c|c|c|}
\hline 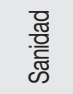 & 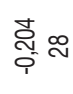 & 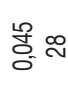 \\
\hline 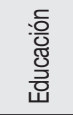 & $\frac{\bar{\sigma}}{\sigma} \stackrel{\sim}{\sim}$ & 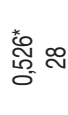 \\
\hline 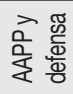 & $\frac{\bar{\sigma}}{\bar{c}} \stackrel{\infty}{\sim}$ & 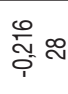 \\
\hline 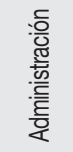 & 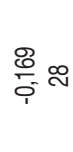 & $\underset{\Xi}{\cong} \stackrel{\sim}{\sim}$ \\
\hline 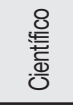 & 矛 & 을 옹 \\
\hline 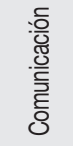 & 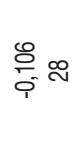 & $\frac{8}{6} \underset{0}{\circ}$ \\
\hline 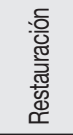 & 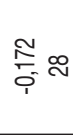 & 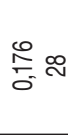 \\
\hline 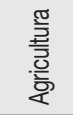 & 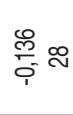 & $\frac{8}{0} \underset{0}{\circ} \stackrel{\sim}{~}$ \\
\hline $\begin{array}{l}\text { 응 } \\
\text { 흥 } \\
0\end{array}$ & 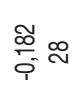 & $\bar{\sigma}_{0} \stackrel{\text { N }}{ }$ \\
\hline 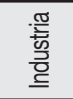 & 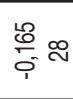 & প్ల్ర \\
\hline \multirow[t]{2}{*}{ 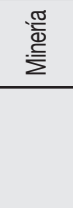 } & 定 & 늉 \\
\hline & 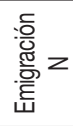 & 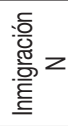 \\
\hline
\end{tabular}

**. La correlación es significativa en el nivel 0,01 (bilateral).

* La correlación es significativa en el nivel 0,05 (bilateral).

Fuente: Elaboración propia. 
Las tasas de desempleo no presentan correlaciones significativas con las tasas de inmigración y de emigración. Se debe a que estas variables están influenciadas por el efecto que la crisis económica ha ejercido sobre el ámbito laboral ya sea en mayor o en menor medida según el país de la UE. Los flujos migratorios han aumentado considerablemente desde el año 2008 en todo el territorio europeo, además, la globalización ha afectado tanto a la mano de obra cualificada como a la no cualificada.

El coeficiente de Pearson en el caso de la correlación entre la inmigración y el PIB pc de los países europeos, muestra una correlación con un valor significativo. Cuanto mayor PIB pc tiene un país europeo, mayor tasa de inmigración experimenta dicho país.

Desde el $\mathrm{FMI}^{6}$ se ha llegado a la conclusión de que un aumento de la inmigración tiene un impacto positivo sobre las sociedades europeas puesto que conlleva un aumento del PIB pc de estas. Esto se debe a que impulsan los niveles de productividad laboral y que, además, el flujo de migrantes mayoritariamente se encuentra en edad laboral, contrarrestando la deficiencia de mano de obra en edad de trabajar derivada del desarrollo demográfico (Europa Press, 2017). Por otro lado, siguiendo un informe de la Oficina Económica del Gobierno, gracias a la entrada de inmigrantes en España, la renta per cápita ha experimentado un impacto neto en torno a un 3\% en los últimos 15 años (Sebastián, 2006).

Con respecto a estas parejas de variables, hay que destacar que la tasa de desempleo y la tasa de emigración no están correlacionadas.

Por otro lado, cuando se observa el valor que el coeficiente de Pearson ofrece al correlacionar la tasa emigración con el número de participantes en el programa Erasmus+, se llega a la conclusión de que, ante un mayor índice de emigración de un país europeo, una menor tasa de participación en el programa Erasmus+.

En el caso de la correlación entre la emigración con la subvención o esfuerzo que efectúan los países europeos, se llega a la misma conclusión que en el caso anterior. Existe una cierta tendencia a aumentar la subvención destinada en el programa Erasmus+ si la tasa de emigración desciende.

${ }^{6}$ Fondo Monetario Internacional. 
Hay países europeos como Alemania, Francia o Reino Unido que invierten gran cantidad de dinero y participan muchos estudiantes universitarios en el programa, cuya tasa de emigración es baja, y que, por el contrario, la de inmigración es mayor. Estos países a su vez coinciden con lo que menor tasa de desempleo presentan, y, por lo tanto, los que mayor oportunidades y facilidades ofrecen a los jóvenes graduados. En este sentido, aunque exista una gran participación en el programa Erasmus+, el talento de los países de origen como los expuestos, no tiende a quedarse en el país destinatario, sino que retornan al finalizar los meses fuera de su país.

Los flujos migratorios no encuentran correlación significativa con el gasto público que los países europeos realizan en términos de educación. El coeficiente que releja la pareja de variables "inmigración - gasto público en educación" es significativo, por lo que se podría decir que una mayor inversión de gasto público en educación conlleva una mayor tasa de inmigración.

Los jóvenes talentos que, o bien están formándose o bien se han graduado recientemente, tienen la oportunidad de continuar con dicha formación e incluso plantearse la posibilidad de emigrar fuera de sus países. Como consecuencia, el destino que la mayoría de los talentos escogerá, serán aquellos que les ofrezcan dichas oportunidades de autodesarrollo personal y profesional, es decir, aquellos países que más inversión realicen en educación y que por lo tanto más opciones oferten a los jóvenes talentos.

Se plantea la cuestión de si una mayor tasa de importaciones o de exportaciones conlleva una mayor o menor tasa de migración. Los resultados nos muestran como no existe correlación entre la inmigración y la capacidad exportadora o importadora de los países de la UE. Una vez más, los datos no resultan los suficientemente significativos como para evaluar esta pareja de variables.

Para llevar a cabo el análisis estadístico entre los flujos migratorios y los graduados (en términos relativos) por niveles educativos, se han tenido en cuenta para evitar posibles desviaciones, veinte países de la UE de los cuales se poseen todos los datos para el año 2015.

En relación con estas parejas de variables, tan solo se llega a una conclusión clara, y es que, a mayor tasa de emigración, menor número de doctores habrá. Esta relación se puede explicar aten- 
diendo a que una mayor tasa de emigración implica una menor renta per cápita y por lo tanto una menor capacidad adquisitiva por parte de la población de afrontar unos estudios postobligatorios que a su vez exigen gran dedicación temporal y económica. También es necesario considerar la posibilidad del joven talento de un país de emigrar ante las pocas becas y oportunidades que un país ofrece para cursar sus estudios de postgrado. Hay que tener en cuenta que este tipo de estudios es voluntario y vocacional, pero que en la mayoría de los casos es requisito indispensable para cubrir las necesidades que las organizaciones solicitan.

Con respecto a las ofertas de empleo en los distintos sectores industriales, el coeficiente más representativo es el del sector educativo, con signo positivo y por lo tanto pudiendo considerarse que una mayor tasa de inmigración conlleva una mayor oferta de empleo en dichos sectores.

\section{CONCLUSIONES}

A lo largo de este estudio, se ha analizado la influencia de distintas variables en el contexto actual de la migración poblacional y de talento en la UE. Por un lado, se ha realizado un intensivo estudio bibliográfico en el que se ha puesto de relieve la importancia de este fenómeno denominado "brain drain", así como las causas que lo originan. Una vez fundamentada la parte teórica, se han recopilado y analizado datos desde dos perspectivas, correlacionándolos para ver la tendencia que siguen. Las conclusiones se pueden estructurar en cuatro puntos:

- Los comportamientos actuales de búsqueda de empleo están tomando cada vez más un aliento globalizado, y en particular, en la UE, los solicitantes de empleo prácticamente no detectan ninguna barrera legal a la migración. La creación de un mercado único que engloba a todos los Estados Miembros, incentiva y potencia los flujos migratorios. Por otro lado, las organizaciones y los países se ven obligados, ante la creciente competencia que conlleva la globalización, a iniciar una guerra de captación de talento, que no solo aporte conocimientos, sino también habilidades, disponibilidad geográfica y disponibilidad temporal. 
- La crisis económica y financiera ha sido el factor fundamental y decisivo para que la población habitante del territorio europeo, ante las escasas oportunidades de empleo y autodesarrollo personal y profesional, haya tomado la decisión de migrar de sus países de origen buscando mayores y mejores ofertas de empleo y formación.

- Las causas fundamentales que originan la fuga de cerebros son la falta de oportunidades de empleo, la existencia de un ambiente social y laboral conflictivo y la falta de apoyo a los estudiantes recién graduados.

- La parte práctica de este proyecto ha puesto de manifiesto seis correlaciones de significación estadística. En primer lugar, una mayor tasa de inmigración conlleva un mayor PIB pc en la UE. También un mayor gasto público en educación implica una mayor tasa de inmigración. Con referencia a la tasa de emigración, cuanto mayor es, menores cifras de participación en el programa Erasmus+ así como en la cuantía destinada al mismo. También trae consigo una mayor tasa de emigración, un menor número de doctores. En relación con las tasas de ofertas de trabajo por sectores de actividad, una mayor tasa de inmigración está positivamente correlacionada con las ofertas del sector educativo.

En conclusión, las asimetrías generadas entre los Estados Miembros como consecuencia de la migración del talento deberán ser valoradas. Por tanto, desde una perspectiva individual, todos los países interesados, deberán ser capaces de generar estrategias de retención de talento para lograr mantener su competitividad, así como desde una perspectiva generalista, la UE deberá ser consciente de los nuevos retos a los que se enfrenta. Sin embargo, este trabajo no está exento de limitaciones. Se deberá realizar un estudio posterior más sofisticado estadísticamente que tenga capacidad explicativa de las relaciones establecidas en este. Además, se podrá replicar el estudio en otros ámbitos y también actualizar los datos para ver la situación actual una vez se ha superado la fase más crítica de la crisis. Este artículo puede servir como primer paso para la realización de futuras investigaciones proporcionando un panorama general de la migración del talento 
en Europa, así como su interrelación entre las variables macroeconómicas y educativas.

\section{BIBLIOGRAFÍA}

Appleyard, R. T. (1992). Migration and Development: A Global Agenda for the Future (Vol. 30). Nedlands, Australia: International Organization of Migration (IOM).

BGRS. (2016). Global Mobility Trends. Recuperado el 15 de septiembre de 2018, de http://globalmobilitytrends.bgrs.com/

Brandi, M. (2006). La historia del brain drain. Revista iberoamericana de ciencia tecnología y sociedad, 3(7), 65-85.

Cabré, A., \& Domingo, A. (2002). Flujos migratorios hacia Europa: actualidad y perspectivas. Arbor, CLXXII (678), 325-344.

Canales Cerón, A. I., \& Zlolniski, C. (2001). Comunidades transnacionales y migración en la era de la globalización. Notas de población.

Chorro, J. L. (2016). Estadística aplicada a Psicología. Recuperado el 20 de Abril de 2017, de Estadística aplicada a Psicología: http://www.uv.es/webgid/Descriptiva/31_coeficiente_de_ pearson.html

De Haas, H. (2010). Migration and development: A theoretical perspective 1. International migration review, 44(1), 227-264.

Delgado, L. (2002). La inmigración en Europa: realidades y políticas. Madrid: Universidad Rey Juan Carlos. Unidad de Políticas Comparadas (CSIC).

Edwards, S. (2001). Capital mobility and economic performance: are emerging economies different? (No. w8076). National bureau of economic research.

Europa.eu. (2016). La historia de la Unión Europea. Obtenido de La historia de la Unión Europea: http://europa.eu/european-union/ about-eu/history_es

Europa Press. (2017). La inmigración aumenta el PIB per cápita en las economías avanzadas, según el FMI. Recuperado el 30 de abril de 2017, de La inmigración aumenta el PIB per cápita en las economías avanzadas, según el FMI: http://www.europapress. es/internacional/noticia-inmigracion-aumenta-pib-per-capitaeconomias-avanzadas-fmi-20170109134323.html 
Hall, D. T., \& Moss, J. E. (1998). The new protean career contract: Helping organizations and employees adapt. Organizational dynamics, 26(3), 22-37.

Haque, N. U., \& Kim, S. J. (1995). "Human capital flight": Impact of migration on income and growth. Staff Papers, 42(3), 577-607.

Harris, J. R., \& Todaro, M. P. (1970). Migration, unemployment and development: a two-sector analysis. The American economic review, 60(1), 126-142.

Kahanec, M. (2012). Labor mobility in an enlarged European Union (Vol. No 6485): Discussion Paper series, Forschungsinstitut zur Zukunft der Arbeit.

Krishnan, S., \& Kirubamoorthy, G. (2017). Cultural Intelligence and Cross-Cultural Adjustments: Impact on Global Mobility Intentions. American Journal of Economics, 7(1), 25-28.

Lewin, A. Y., Massini, S., \& Peeters, C. (2009). Why are companies offshoring innovation? The emerging global race for talent. Journal of International Business Studies, 40(6), 901-925.

Mahroum, S. (2000). Highly skilled globetrotters: mapping the international migration of human capital. R \& D Management. Blackwell Publishers.

Miyagiwa, K. (1991). Scale economies in education and the brain drain problem. International Economic Review, 743-759.

OCDE. (2008). The Global Competition for Talent: Mobility of the Highly Skilled.

Papademetriou, D., \& Martin, P. (1991). The Unsettled Relationship: Labor Migration and Economic Development. Greenwood Press.

Pellegrino, A. (2000). Drenaje, movilidad, circulación: nuevas modalidades de la migración cualificada. Comisión Economica para América Latina (CEPAL), Organización Internacional para las Migraciones.

Puri, L. (2008). Contribución del comercio al fomento del desarrollo y la reducción de la pobreza: movilidad laboral e intercambio de trabajadores. Naciones Unidas.

Savage, M., \& Egerton, M. (1997). Social mobility, individual ability and the inheritance of class inequality. Sociology, 31(4), 645-672. 
Sebastián, M. (2006). Inmigración y economía española: 19962006. Recuperado el 30 de abril de 2017, de Inmigración y economía española: 1996-2006: http://mcaugt.es/documentos/0/ doc1501.pdf

Shachar, A. (2006). The race for talent: Highly skilled migrants and competitive immigration regimes. NYUL rev., 81, 148.

South, S. J., \& Deane, G. D. (1993). Race and residential mobility: Individual determinants and structural constraints. Social forces, 72(1), 147-167.

Templer, K. J., Tay, C., \& Chandrasekar, N. A. (2006). Motivational cultural intelligence, realistic job preview, realistic living conditions preview, and cross-cultural adjustment. Group \& Organization Management, 31(1), 154-173.

\section{INSTITUCIONES Y BASES DE DATOS}

Base de datos macroeconómica anual de la Dirección General de Asuntos Económicos y Financieros de la Comisión Europea (AMECO). Página web: https://ec.europa.eu/info/businesseconomy-euro/indicators-statistics/economic-databases/ macro-economic-database-ameco_en

Comisión Europea Erasmus +. Página web: https://ec.europa.eu/ programmes/erasmus-plus/node_es

Comisión Europea: Empleo, Asuntos Sociales e Inclusión. Página web: http://ec.europa.eu/social/main.jsp?catld=157\&langld=es

Eurostat. Página web: http://ec.europa.eu/eurostat

Instituto Nacional de Estadística (INE). Página web: http://www. ine.es/

Organización para la Cooperación y el Desarrollo Económico (OCDE). Página web: http://www.oecd.org/ 